ISSN: 1988-2688

http://revistas.ucm.es/index.php/RCCV

http://dx.doi.org/10.5209/rev_RCCV.2015.v9.n2.51041

Revista Complutense de Ciencias Veterinarias 2015 9(2):22-40

\section{R C C V}

Revista Complutense de Ciencias Veterinarias

\title{
CONGELACIÓN DE EMBRIONES BOVINOS
}

\section{EMBRYO FREEZING CATTLE}

Alejandro Córdova Izquierdo ${ }^{1 *}$, Juan Eulogio Guerra Liera ${ }^{2}$, Abel Villa Mancera ${ }^{3}$, Jaime Olivares Pérez $^{4}$, Gerardo Cansino Arroyo ${ }^{5}$, Ma. De Lourdes Juárez Mosaqueda ${ }^{6}$ y José Félix Pérez Gutiérrez $^{7}$

${ }^{1}$ Departamento de Producción Agrícola y Animal. Universidad Autónoma Metropolitana Unidad Xochimilco, México, D.F.. ${ }^{2}$ Facultad de Agronomía. Universidad Autónoma de Sinaloa, México.

${ }^{3}$ Facyultad de Veterinaria. Benemérita Universidad Autónoma de Puebla, México. ${ }^{4}$ Unidad Académica de Veterinaria Ciudad Altamirano. Universidad autónoma de Guerrero, México. ${ }^{5}$ División Académica de Ciencias agropecuarias. Universidad Juárez Autónoma de Tabasco, México. ${ }^{6}$ Departamento de Morfología. FMV-UNAM, México.

${ }^{7}$ Facultad de Veterinaria. Universidad Complutense de Madrid, España.

*correspondencia: acordova@correo.xoc.uam.mx

\section{RESUMEN}

La congelación de embriones bovinos es una biotecnología aplicada a la Reproducción Animal de esta especie, cuyos beneficios es ya una realidad. El objetivo de este trabajo es mostrar aspectos de gran importancia para la congelación de embriones bovinos. En el cual se describen los siguientes puntos: Definición de congelación, beneficios de la congelación de embriones bovinos, desventajas de la congelación de embriones bovinos, variables a tomarse en cuenta para el éxito de la congelación de embriones bovinos, crioprotectores, funciones de un crioprotector, métodos de conservación de embriones bovinos, procedimientos convencionales en la congelación lenta de embriones, etiquetado de pajillas en la congelación de embriones bovinos, técnicas de descongelación de embriones, método One-step y crioinjuria. 
PALABRAS CLAVE: Congelación, embriones, bovinos, crioprotectores.

\begin{abstract}
.
The freezing of bovine embryos is a biotechnology applied to animal reproduction of this species, whose benefits is already a reality. The aim of this paper is to show aspects of great importance for freezing bovine embryos. Definition of freezing, freeze benefits of bovine embryos, freezing disadvantages of bovine embryos, variables to be taken into account for the success of the freezing of bovine embryos, cryoprotectants, a cryoprotectant functions: where the following described, conservation methods bovine embryos, conventional methods in the slow freezing of embryos, labeling straws in the freezing of bovine embryos, embryo thawing techniques, and crioinjuria One-step method.
\end{abstract}

KEY WORDS: Freezing embryos, cattle, cryoprotectants

\title{
INTRODUCCIÓN
}

En la actualidad, algunas de las biotecnologías de reproducción animal asistida que se conocen son una realidad, especialmente en el ganado bovino; cuyo interés por parte de profesionales y productores se hace más evidente cada día en todo el mundo, tal es el caso de la producción de embriones, tanto in vitro como in vivo y dentro de ésta biotecnología, la conservación de embriones mediante la congelación es un tema de vital trascendencia.

La producción de embriones bovinos producto de fecundación in vitro o in vivo y la superovulación de vacas de alto valor genético, son herramientas que ofrecen nuevas perspectivas para la aceleración del progreso genético en la ganadería, tanto lechera como de carne. Se sabe hace más de 43 años que la congelación de embriones para su conservación y posterior utilización es una técnica esencial, que tiene como objetivo almacenar los embriones por largos periodos de tiempo y que posteriormente puedan ser utilizados con éxito

La utilización de las bajas temperaturas para conservar los embriones producidas por estas biotecnologías se ha convertido en una herramienta indispensable que permitirá consolidar 
y aumentar el impacto de estas biotecnologías en la ganadería de cualquier país del mundo; de tal manera que la conservación de embriones bovino mediante la congelación presenta varias ventajas, desde el punto de vista biológico como comercial, las cuales pueden resumirse de la siguiente manera (Akyurt et al., 2002; Díez, 2003; Ochoa, 2011):

1. Permite una reducción de costes derivados de la aplicación de las tecnologías reproductivas.

2. Facilita una disociación de todas estas técnicas de la actividad reproductivas cíclica que presentan las especies mamíferas de interés, consiguiéndose una independencia temporal del estado fisiológico de los animales (hembras receptoras, por ejemplo).

3. Ayuda a evitar los desvíos genéticos, es decir evita que la genética se vaya propagando hacia una fuente no deseada.

4. Ayuda a eliminar patologías que normalmente se asocian al mantenimiento de animales vivos.

5. Posibilita la conservación de razas o especies en riesgo de extinción mediante la creación de bancos de embriones congelados, manteniendo intacto el patrimonio genético.

El éxito en la congelación de embriones bovinos depende de varios factores; sin embargo, lo que persiguen los principios fundamentales de la congelación es la protección celular frente a los principales efectos perjudiciales del proceso; tales como formación de hielo intracelular, deshidratación y efecto tóxicos de los crioprotectores.

En la actualidad existen metodologías para congelar embriones bovinos, estas nos permiten la aplicación comercial de biotecnología para embriones bovinos producidos in vivo. Considerando que, en general, el promedio de las vacas donantes es de aproximadamente 8 a 10 ovocitos recolectados, que de ellos se obtiene 6 a 7 embriones transferibles, dando 3 a 4 gestaciones exitosas y que la tasa de preñez es del orden de $60 \%$ utilizando embriones frescos y 30 a $40 \%$ con embriones criopreservados, la potencialidad exponencial que tiene este método de reproducción asistidad ligra maximizar la explotación de la espcie bovina (Belascoain et al., 2010).

\section{DEFINICIÓN DE CONGELACIÓN}

La criopreservación tiene como objetivo el mantenimiento de la viabilidad y funcionalidad celular a temperaturas bajas (Ávila et al., 2006). La congelación es un proceso 
mediante el cual las células o tejidos se conservan a bajas temperaturas, generalmente entre - 80 y $-196^{\circ} \mathrm{C}$, ésta última temperatura conocida como el punto de ebullición del nitrógeno líquido, se usa con el fin de disminuir las funciones vitales y de esta manera poder mantener a dichas células o tejidos en condiciones de vida latente suspendida por tiempo indefinido (Maurer, 1978; Niemann, 1985; Ávila-Portilla et al., 2006; Hincapie et al., 2005; Ochoa, 2011). Durante el proceso de congelación, las células embrionarias sufren cambios importantes asociados a la formación de hielo intra o extracelular que pueden disminuir la sobrevida poscriopreservacion. Para poder minimizar estos problemas, se ha recurrido al uso de "sustancias crioprotectoras", así como a tasas de descenso térmico controladas mediante la utilización de máquinas congeladoras programables de alto costo (Mucci et al., 2005).

\section{BENEFICIOS DE LA CONGELACIÓN DE EMBRIONES BOVINOS}

Económicos:

Las ventajas de la congelación de embriones bovinos, son de tipo económico y zooténicos. Desde el punto de vista económico, todos los esfuerzos que se realicen para mantener la calidad de los recursos genéticos de los bovinos, quedan justificados. En primer lugar, contribuye al mantenimiento de muchos ecosistemas y en segundo lugar, a la optimización de los costos en el transporte de material genético de alta calidad entre cualquier parte del mundo; por ejemplo, con el costo del transporte de un animal vivo, se podrían transportar hasta cerca de 4 mil embriones congelados.

Zootécnicas:

Las ventajas zootécnicas de la congelación de embriones bovinos, está reflejada en el progreso genético rápido que representa el utilizar embriones congelados-descongelados en la reproducción de bovinos especializados en la producción de elche como en la de carne, en cualquier parte del mundo.

Beneficios operativos sobre los frescos:

Los beneficios operativos que representan los embriones congelados de bovinos sobre los frescos, se presentan a continuación:

- Se aprovecha todo los embriones en de una colecta, sobre todo cuando no se dispone de suficientes receptoras para su transferencia en fresco. 
- Pueden realizarse transferencias simultáneas de embriones de diferentes combinaciones de padre. Esto es importante para las exposiciones y para las pruebas de comportamiento; debido a la disponibilidad de embriones en cualquier momento.

- La disponibilidad de embriones congelados en las unidades de producción bovina para la reproducción, ayuda la programación de las nacencias de acuerdo a la capacidad de la Unidad de producción.

- Con la disponibilidad de embriones congelados, ayuda a trabajar con lotes más pequeños de receptoras, ya que los embriones congelados pueden utilizarse en cualquier momento que se requiera.

Comerciales:

- Los embriones de bovinos congelados, cuyo proceso de congelación ha seguido las normas de higiene y sanidad de la Sociedad Internacional de Transferencia de Embriones de tal manera no transmiten ninguna enfermedad, desapareciendo las barreras sanitarias, cuyo beneficio es el comercio en todo el mundo de embriones congelados de bovinos.

- Los embriones de bovinos congelados, pueden ser descongelados y transferidos en cualquier época del año y lugar geográfico a receptoras adaptadas a los factores ambientales de un lugar determinado de cualquier parte del mundo.

- Con el uso de embriones bovinos congelados se disminuye el problema de adaptación a elevadas temperaturas, altitud, ectoparásitos moscas y garrapatas por ejemplo, etc. Obteniendo de esta manera una genética adaptada en cualquier parte del mundo.

\section{DESVENTAJAS DE LA CONGELACIÓN DE EMBRIONES BOVINOS (Ochoa, 2011).}

Afortunadamente, son pocas las desventajas que representa la congelación de embriones bovinos; en la actualidad ya son pocas, se presentan a continuación:

- El índice de gestación obtenido con embriones congelados es $10 \%$ inferior al de embriones frescos.

- De tipo financiero, se debe esperar como mínimo dos años para que los animales nacidos de embriones comiencen a producir. 


\section{VARIABLES A TOMARSE EN CUENTA PARA EL ÉXITO EN LA CONGELACIÓN DE EMBRIONES BOVINOS}

Las variables que deben tomarse en cuanta en la congelación de embriones bovinos, se presentan a continuación (Maurer, 1978; Niemann, 1985; Ávila-Portilla et al., 2006; Hincapie et al. 2005; Ochoa, 2011):

- Tipo y concentración de los compuestos crioprotectores.

Ya que existen una amplia variedad de componentes semejantes, que han demostrado proteger a las células, de los daños que puedan sufrir por la congelación. Estos componentes son usualmente divididos en dos categorías, los que permiten penetrar o proteger a las células (Glicerol, Dimetil, Sulfoxido y el Etil Glico) y los no penetrantes a las células (sacarosa, polivinilpirolidona, hidroxietil almidón HES, Dextrano, Albúmina). Los no penetrantes como la sacarosa, mantienen una alta presión osmótica en el medio extracelular durante la remoción de crioprotector. Esto previene el shock osmótico, debido a la difusión del glicerol fuera del embrión después de la descongelación.

Tipos

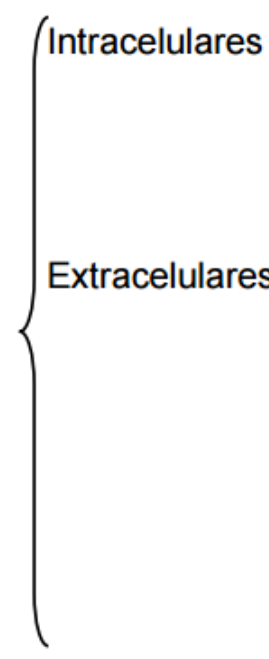

Características
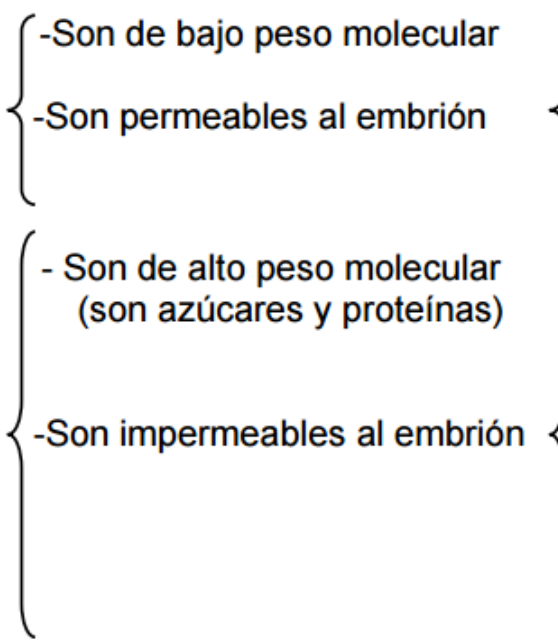

\section{Ejemplos}

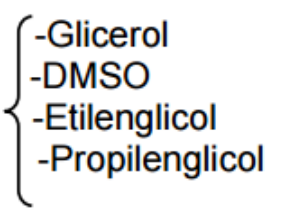

$\left\{\begin{array}{l}\text {-Sucrosa } \\ \text {-Seroalbumina bovina } \\ \text {-Ácido hialurónico } \\ \text {-Polivinilpirrolidona(PVP) } \\ \text {-Hidrosietilo de almidón (HES) } \\ \text {-Dextranos } \\ \text {-1,2 propanadiol (PROH) } \\ \text {-Glucoproteínas de peces del } \\ \text { antártico (congelan a }-2.5^{\circ} \mathrm{C} \text { ) }\end{array}\right.$

Figura 1. Tipos de compuestos crioprotectores, características y ejemplos (modificado de: Rivas y Barceló, 2011)

- Formación de hielo.

El punto de congelación de una solución depende del número de moléculas de soluto presente. Esto significa que el hielo no se forma en estas soluciones hasta que su enfriamiento 
baje a su respectivo punto de congelación. Solamente, dependiendo de la extensión del volumen congelado existente, soluciones acuosas exhiben un comportamiento no estable, donde la súper congelación baja el verdadero punto de congelación, sin formación de hielo. Este fenómeno de súper congelación puede jugar un papel muy importante en la supervivencia de las células que comienzan a congelarse, especialmente los embriones y ovocitos de mamíferos, ya que estas células tienen un comportamiento osmótico específico.

- Ritmo de enfriamiento de $-5 \mathrm{a}-70{ }^{\circ} \mathrm{C}$.

Las células deben ser enfriadas a tasas altas o bajas que garanticen su supervivencia, a la congelación y a la descongelación. Afortunadamente existen tasas óptimas de enfriamiento con un alto porcentaje de supervivencia a muchos tejidos de células. Dependiendo el coeficiente de permeabilidad de las células al agua y el coeficiente de temperatura de los tipos de células, estas se van a tener una tasa óptima de enfriamiento.

- Temperaturas de conservación.

En general se utilizan dos temperaturas, $-80^{\circ} \mathrm{C}$ y $-196^{\circ} \mathrm{C}$. Las células pueden ser preservadas por largo tiempo en depósito a $-130^{\circ} \mathrm{C}$, temperatura de transición de cristal de hielo. La forma de extender el tiempo y la seguridad del depósito de células congeladas es introducirlas en el líquido $\left(-196^{\circ} \mathrm{C}\right)$; o en vapor $\left(-150^{\circ} \mathrm{C}\right)$ del nitrógeno líquido.

- Ritmo de descongelación de -70 a $-5{ }^{\circ} \mathrm{C}$ a $36^{\circ} \mathrm{C}$.

El tiempo de congelación a $-196^{\circ} \mathrm{C}$, de un embrión, el cual ha sido enfriado a $0,2^{\circ} \mathrm{C} / \mathrm{min}$, es muy diferente, que uno que ha sido enfriado a $2^{\circ} \mathrm{C} / \mathrm{min}$. Si la tasa de calentamiento es moderada, el embrión, aparentemente, puede permanecer en equilibrio osmótico, cuando es calentado. Si la tasa es alta, el embrión puede sufrir una rehidratación larga o rápida durante el calentamiento. Aparentemente si la rehidratación es muy baja y rápida, el embrión muere (Figura 2). 


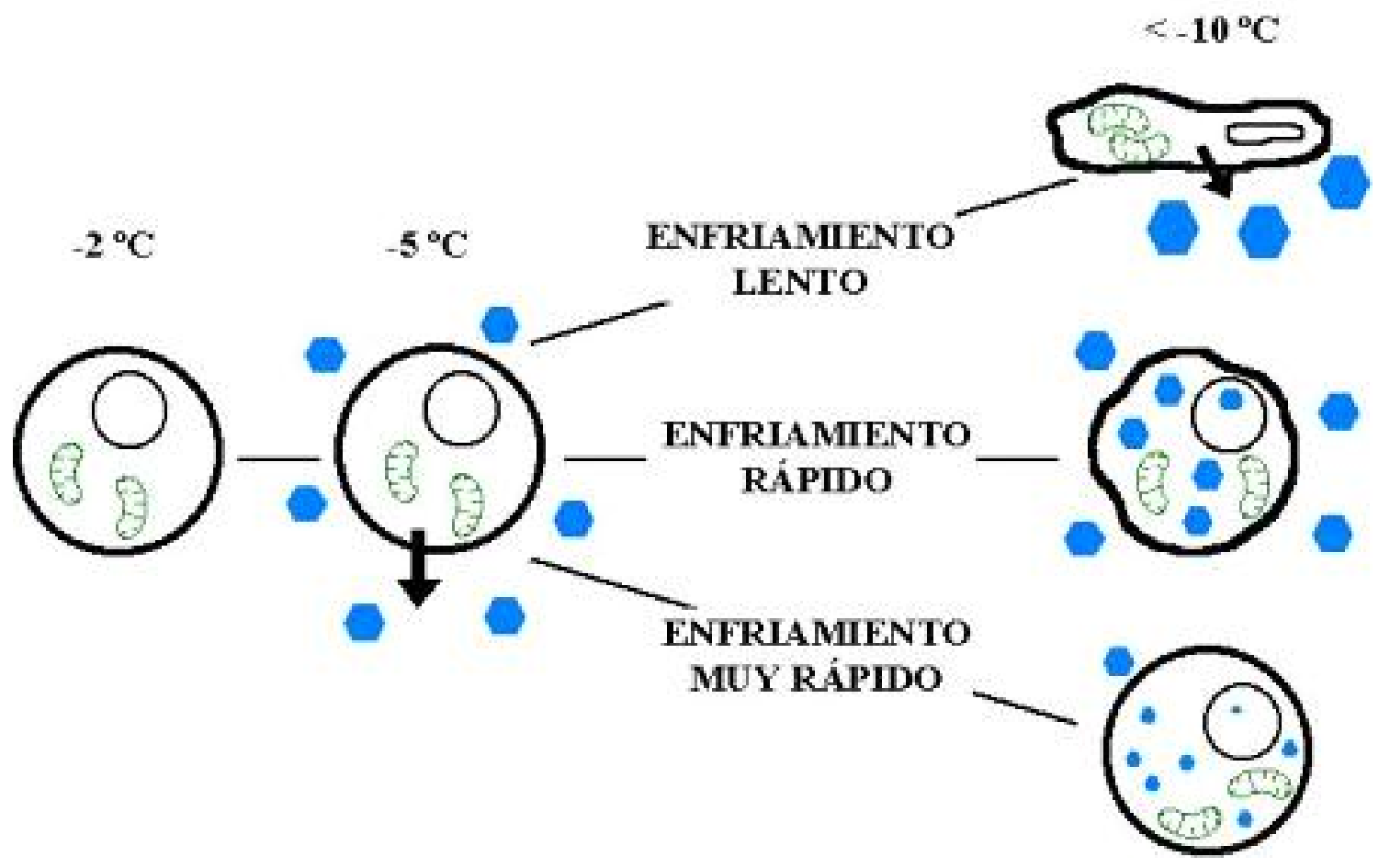

Figura 2. Figura que representa las situaciones que pueden ocurrir durante el proceso de congelación de embriones bovinos (Adaptado de Mazur, 1978).

- Grado de dilución y temperatura.

Se refiere a la tasa y temperatura a la cual la célula del embrión es diluida fuera de la disolución en la cual, fue originalmente congelada. Con embriones congelados hay que tener mucho cuidado con la dilución de los componentes protectores, en forma semejante, para evitar el choque osmótico. El choque puede ocurrir, si el embrión tiene una alta tasa de concentración de solutos protectores y es de repente, transferido a una solución isotónica (Noris et al., 1998).

\section{CRIOPROTECTORES}

Los crioprotectores son sustancias que protegen a la célula cuando es sometida a bajas temperaturas y son necesarios en las soluciones de congelación para prevenir el daño celular durante la congelación y descongelación de embriones bovinos, se conocen dos tipos que a continuación se mencionan (Maurer, 1978; Niemann, 1985; Ávila-Portilla et al., 2006; Hincapie et al. 2005; Ochoa, 2011):

1. No penetran (impermeables, extraceluares) de alto y bajo PM. 
2. Penetran (Permeables, intracelulares) de bajo PM.

En seguida se presentan algunos ejemplos:

No penetran (impermeables, extraceluares):

1. De bajo PM: Glucosa, Sacarosa y otros azucares.

Deshidratan las células antes de la congelación minimizando la formación de cristales.

Estabilizan la estructura de la membrana.

2. De alto PM: polivinilpirrolidona (PVP), glucosa, fructosa, ficol, dextrano, sorbitol, sucrosa, lactosa. Trealosa, rafinosa,

Protegen en congelación/descongelación alterando el tamaño y la forma de los cristales.

Penetran (Permeables, intracelulares):

Bajo PM: Glicerol, Dimetilsulfóxido (DMSO), 1-2 Propanodiol, Etilenglicol (EG), propilenglicol (PG) y etanol.

Reemplazan el agua intracelular minimizando la formación de cristales. También regulan la deshidratación y protegen la estructura proteica.

El peso Molecular de los principales crioprotectores utilizados en la congelación de embriones bovinos, se presenta en la siguiente tabla:

\begin{tabular}{cc}
\hline Crioprotector & Peso Molecular \\
\hline Etilenglicol (EG) & 62.07 \\
\hline Propilenglicol (PG) & 76.10 \\
\hline Dimetil sulfóxido (DMSO) & 78.13 \\
\hline Glicerol & 92.10
\end{tabular}

Tomado y adaptado de: Maurer, 1978; Niemann, 1985; Ávila-Portilla et al., 2006; Hincapie et al., 2005; Ochoa, 2011.

\section{FUNCIONES DE UN CRIOPROTECTOR}

- Previenen la deshidratación total y la degeneración proteica, causada por la congelación del agua intra y extracelular durante el proceso.

- No deben ser tóxicos a los embriones. 
NOTA: Para reducir el daño osmótico y tóxico del crioprotector, debido a la alta concentración de sales, se ha dejado de utilizar G y DMSO para hacer uso de EG, solo o en combinación con sucrosa.

EG tiene mayor velocidad de penetración y necesitaría menor tiempo de exposición, disminuyendo su efecto tóxico.

- Existe relación directa con el estado de desarrollo embrionario y la velocidad de penetración del crioprotector.

- La permeabilidad de los embriones a los crioprotectores se incrementa después de la fecundación, aumentando a medida que el desarrollo embrionario progresa por la diferencia que existe en la relación área/volumen.

- Embriones producidos in vitro tienen menor tolerancia a la congelación debido a la formación de hielo intracelular, aumento en la concentración de lípidos y enzimas que digieren la zona pelúcida, lo que los hace más sensibles al enfriamiento y a la invasión de virus, bacterias y hongos.

- La calidad de los embriones a congelar es de vital importancia, ya que cuando se congelan embriones de buena calidad, se obtienen mejores resultados que cuando se congelan de calidad regular.

- La congelabilidad de los embriones mamíferos varía con la especie del animal.

\section{MÉTODOS DE CONSERVACIÓN DE EMBRIONES BOVINOS}

Existen cuatro métodos para la conservación de embriones bovinos, los cuales se presentan a continuación; sin embargo, en este trabajo solo se describe el procedimiento del tercero.

1. Temperatura ambiente.

2. Refrigeración.

Los embriones en largo tiempo y a temperatura ambiente decrecen su capacidad de desarrollo. Por medio de la refrigeración a temperaturas de 0 a $4^{\circ} \mathrm{C}$ por no más de 24 horas. Se puede mantener a los embriones en PBS envasados en pajuelas de $0.25 \mathrm{ml}$ y colocadas en un refrigerador se utiliza hielo y agua para regular el descenso de la temperatura. La refrigeración de embriones puede ser considerada como una alternativa interesante cuando no es posible recurrir a la congelación. No obstante, a pesar de los buenos resultados y de su sencillez, en la actualidad 
ha caído prácticamente en desuso debido a que la congelación convencional se ha convertido en una técnica que si bien es más costosa, permite mantener la viabilidad embrionaria por tiempos limitados con las ventajas prácticas que ellos trae aparejado (Ochoa, 2011).

3. Congelación.

El proceso de congelación podría resumirse mencionando que a medida que una solución acuosa se enfría por debajo de $0^{\circ} \mathrm{C}$ disminuye el movimiento molecular, sobre enfriándose hasta alcanzar una masa crítica de núcleos, momento en el cual se libera el calor latente de cambio de estado. Uno de los efectos de la cristalización de las colusiones acuosas es remover el agua de la solución, ya que los cristales que se forman estas compuestos por agua pura, por lo cual la solución remanente que permaneces sobreenfriada, se concentran. De este modo, esta nueva solución permanece liquida hasta que alcanza, mediante una mayor extracción de calor, el nuevo punto de equilibrio de congelamiento. Este proceso se repite hasta que una determinada temperatura, denominada eutéctica o "punto eutéctico", la fase líquida remanente y los solutos solidifican (Mucci et al., 2005).

\section{Vitrificación.}

El estado vítreo, es aquel alcanzado por el rápido enfriamiento de un medio líquido en ausencia de cristales de hielo y con una elevada viscosidad. Esta solución adquiere un aspecto amorfo similar al vidrio, del cual toma su denominación.

Los factores que afectan la probabilidad de vitrificación de una solución son la viscosidad inicial, su volumen y la tasa de enfriamiento a la cual es sometida. La adición de distintos solutos disminuye la probabilidad de nucleación y crecimiento de los cristales de hielo porque al aumentar la viscosidad, se retrasa el reordenamiento de las moléculas de agua para formar el cristal de hielo dificultándose de este modo los proceso de nucleación y crecimiento y debido a que los solutos son incompatibles con la estructura de hielo, por carecer de una cantidad determinada de agua pura, se torna difícil que los núcleos alcancen el tamaño crítico.

Durante el proceso de vitrificación, el rápido enfriamiento disminuye bruscamente el movimiento molecular, de modo que las moléculas de agua no tienen el tiempo suficiente para ordenarse y orientarse, de acuerdo a sus cargas, para formar los cristales de hielo. Por esta razón las soluciones vitrificadas mantienen la distribución iónica y molecular de un líquido pero en un estado sobreenfriado y extremadamente viscoso, con un aspecto brillante y transparente que lo diferencia del cristalino el cual es opaco y sin brillo. 
Algunas de las ventajas de la vitrificación es que no hay formación de cristales de hielo, mayor rapidez en el procesamiento de cada muestra, y menor costo, por prescindir de máquinas congeladoras programables. Debido a esto, la vitrificación constituye una herramienta interesante capaz de reemplazar a la congelación convencional, especialmente cuando e intenta conservar embriones sensibles a la criopreservación como son los producidos in vitro (Mucci et al., 2005).

\section{PROCEDIMIENTOS CONVENCIONALES EN LA CONGELACIÓN LENTA DE EMBRIONES}

En la congelación de embriones bovinos, deben seguirse los siguientes pasos (Maurer, 1978; Niemann, 1985; Ávila-Portilla et al., 2006; Hincapie et al. 2005; Ochoa, 2011):

- Identificación y clasificación de los embriones.

Antes de someter los embriones a la congelación se debe realizar una rigurosa selección para determinar su calidad, aspecto que va a determinar la posibilidad de sobrevivir a los procesos de congelación y descongelación.

La congelación no debe practicarse en embriones que no sean clasificados con las categorías de bueno o excelentes (Sánchez et al., 2014). La evaluación morfológica de los embriones considera los siguientes criterios sobre la estructura y calidades de un embrión de excelente calidad. Para la evaluación morfológica es necesario tener en cuenta los siguientes aspectos: forma esferoide, simetría de los blastómeros, tonalidad uniforme, estructuras visibles, estado de agregación de las células, variación de talla entre las células, células desprendida en el espacio perivitelino e integridad de la zona pelucida.

De acuerdo a su aspecto morfológico los embriones son categorizados en diferentes grados o escalas de calidad:

Grado 1 (Excelentes): Embriones considerados como normales por su respectivo estado de desarrollo. Muestran contornos celulares bien definidos y no hay dispersión ni destrucción en los blastómeros (Figura 3).

Grado 2 (buenos): Embriones con algunos cambios tales como granulaciones atípicas, algunas con signos de degeneración y opacidades anormales (Figura 4).

Grado 3 (pobre calidad o regulares): Embriones que presentan blastómeros dispersos (poca cohesión) y mayor grado de degeneración ocasionalmente blastómeros asimétricos pero parte de la masa celular se mantiene viable. 
Grado 4 (Malos) alto grado de degeneración que imposibilita determinar el grado de desarrollo (Reggie, 2011).

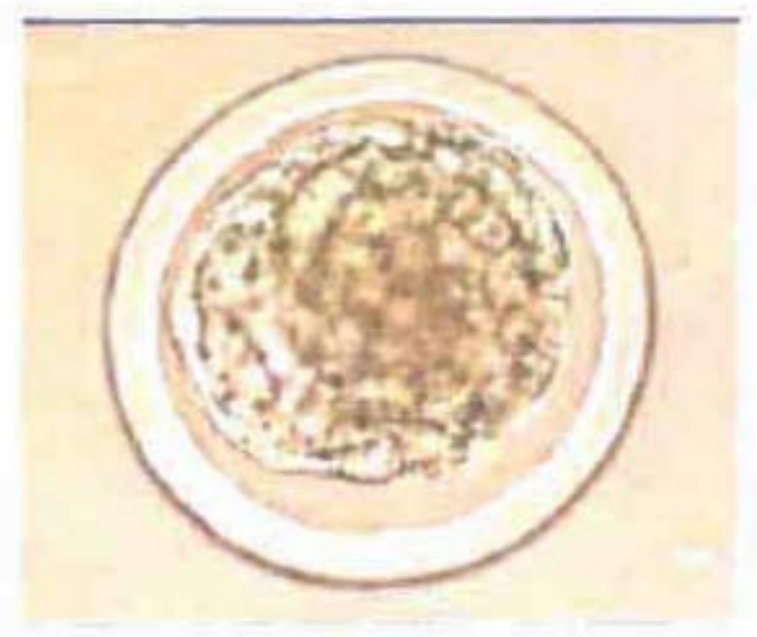

Figura 3. Embrión Grado 1

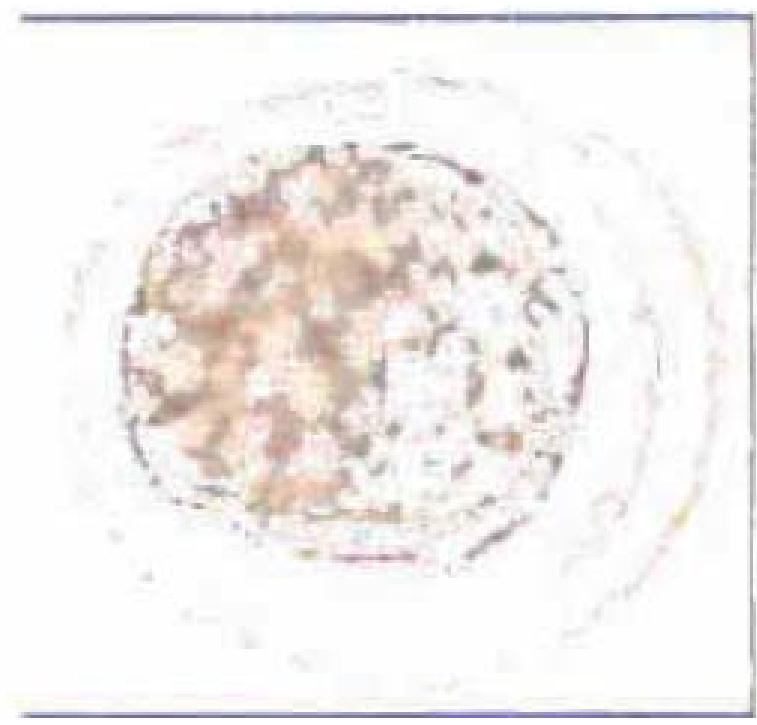

Figura 4. Embrion grado 2.

- Lavado de los embriones según las normas de la Sociedad Internacional de Transferencia Embrionaria (IETS).

El procedimiento de lavado recomendado comprende el transporte de embriones en grupos de 10 o menos, a través de 10 gotas con medios. Se utiliza una micropipeta estéril y nuevo para cada lavado, que deberá constituir una dilución 1:100 con respecto al lavado previo. Los embriones deberán ser agitados ligeramente en cada lavado y tan pronto como se haya efectuado 
esto, se pasara al lavado siguiente. Únicamente los embriones del mismo donante deberán lavarse juntos (Stringfellow y Sidel, 2000).

- Exposición de los embriones a temperatura ambiente $\left(22^{\circ} \mathrm{C}\right)$ a concentraciones: para etilenglicol entre 5 y 10 minutos y para el glicerol entre 10 y 20 minutos.

- Inducción de la formación de cristales (seeding) entre -5 y $-7^{\circ} \mathrm{C}$.

Generalmente la inducción del hielo a los embriones dentro de la pajuela en el medio de crioconservación, se realiza $1{ }^{\circ} \mathrm{C}$ a $2^{\circ} \mathrm{C}$ por debajo del punto de congelación del medio donde se encuentran los embriones para evitar el enfriamiento excesivo. Cuando el programa del equipo de congelación llega a descender la temperatura a $-7^{\circ} \mathrm{C}$ se deja equilibrar la temperatura por espacio de 16 a 20 segundo y se realiza el seeding poniendo en contacto un objeto metálico, (por lo general se utiliza una pinza a temperatura sumamente baja que se logra introduciendo dicho objetivo en nitrógeno líquido), con la superficie de la pajuela pero con la precaución de que el contacto debe hacerse lo más alejado posible de la columna del medio que contiene el embrión y este contacto debe ser estrecho de poca duración ( 1 a 2 segundos) (Sánchez et al., 2014)

- Descenso lento y controlado de la temperatura $\left(0,3\right.$ a $\left.1,0^{\circ} \mathrm{C} / \mathrm{min}\right)$ hasta $\operatorname{los}-30 \mathrm{o}-35^{\circ} \mathrm{C}$.

A medida que baja la temperatura, mayor cantidad de agua puede ser convertida en cristales de hielo, pero igualmente este descenso en la temperatura influye en un descenso en la calidad de agua intracelular. Por consiguiente, el éxito de la criopreservación lenta depende del logro de un equilibrio óptimo entre 1 tasa a la cual el agua abandona la célula y la tasa a la cual esta convertida en cristales de hielo.

El descenso controlado de la temperatura continua a una tasa de 0,2 a $2,0{ }^{\circ} \mathrm{C} / \mathrm{min}$ hasta no producir cambios de volumen en el embrión, ocurriendo esto entre $-30 \mathrm{y} 40{ }^{\circ} \mathrm{C} / \mathrm{min}$, momento en el cual son sumergidos en nitrógeno líquido a $-196^{\circ} \mathrm{C}$ (Cabrera y Fernández, 2006).

- Envasado para la congelación de embriones bovinos

Actualmente el elemento más utilizado para contener los embriones, tanto para la criopreservación como para su transporte y/o transferencia a una hembra receptora, lo constituye la pajuela de plástico de $0,25 \mathrm{ml}$ de capacidad. Existen diversos modos de cargar las pajuelas, pero en la mayoría, el o los embriones se encuentran contenidos en una columna de 0,5 a 2 centímetros de solución de congelación separado de otras columnas, ya sea de PBS con suero o 
soluciones con sacarosa como el método One-Step (Se describe más adelante a detalle), separadas por burbujas de aire (Ochoa, 2011).

- Descongelación controlada alrededor de $250^{\circ} \mathrm{C} / \mathrm{min}$ (Descrito más adelante).

- Remoción del crioprotector a temperatura ambiente (en el caso del G).

Luego de la congelación-descongelación, la mayoría de los agentes crioprotectores deben ser extraídos de los embriones antes de ser trasferidos a hembras receptoras o colocados en cultivo. Esto debe efectuarse si al momento de la descongelación, las células cargadas de crioprotectores son expuestas, por ejemplo, a una solución de PBS isosmolar respecto de las células en condiciones normales o al tracto reproductor femenino, debido a que el agua se difunde más rápido que estos compuestos a través de la membrana plasmática, las células se desintegran por el shock osmótico. Por este motivo, se recurre a la rehidratación del embrión mediante pasajes a través de concentraciones y osmolaridades decrecientes del crioprotector utilizado. Otra alternativa es emplear sustancias no penetrantes como la sacarosa, cuya osmolaridad sea igual o ligeramente inferior a la alcanzada con el crioprotector penetrante utilizado, lo cual disminuye la entrada de agua mientras se produce la salida del crioprotector penetrante (Ochoa, 2011).

- Transferencia del embrión a una receptora.

\section{ETIQUETADO DE PAJILLAS EN LA CONGELACIÓN DE EMBRIONES BOVINOS}

Según los lineamientos de la Sociedad Internacional de Transferencia de embriones, en el etiquetado para la congelación de embriones bovinos, se debe seguir los siguientes 12 pasos (Maurer, 1978; Niemann, 1985; Ávila-Portilla et al., 2006; Hincapie et al. 2005; Ochoa, 2011):

1. Número de la pajilla (1)

2. Tipo de solución que se utilizó para el congelamiento. Por ejemplo DT: si es en congelado en etilenglicol.

3. Número del laboratorio que realiza T.E. (registro: 1279).

4. Raza (AN: Angus).

5. Número de registro de la vaca (14763076).

6. Nombre de la vaca (N256).

7. Raza de la vaca (AN: Angus).

8. Número de registro del toro (14542822).

9. Nombre del toro (opcional, por ejemplo SUM. PFRED ON12). 
10. Número de embriones.

11. Grado y calidad del embrión.

12. Fecha de colecta y congelamiento.

Ejemplo:

1 DT 1279 AN 14763076 N256 AN 14542822 SUM. PFRED ON12 1 EMB. 4-1 07MR14

\section{TECNICAS DE DESCONGELACION DE EMBRIONES}

Para la descongelación de los embriones bovinos, se debe tomar en cuenta los siguientes cuatro pasos generales (Maurer, 1978; Niemann, 1985; Ávila-Portilla et al., 2006; Hincapie et al. 2005; Ochoa, 2011):

1. La descongelación puede realizarse exponiendo las pajillas a temperatura ambiente durante 10 segundos antes de colocarlas en baño María 30-35 ${ }^{\circ} \mathrm{C}, 20-30$ segundos.

2. Los crioprotectores pueden ser removidos de los embriones en tres y seis pasos en forma escalonada, hasta lograr una concentración final de PBS sin crioprotector, utilizando dos pasos con sucrosa $0.5 \mathrm{M}$ y $0.25 \mathrm{M}$, y manteniendo al embrión durante cinco minutos en cada una (complicada).

3. El embrión también puede transferirse en forma directa.

4. Esta técnica permite transferir embriones congelados a campo sin necesidad de equipos y laboratorios muy costosos, obteniendo tasas de preñez que oscilan entre el 30 y el $50 \%$.

\section{METODO ONE-STEP}

El método" One-Step" representa una gran ventaja práctica dado que posibilidad transferir embriones congelados a campo de manera similar a lo que ocurre con la inseminación artificial, no obstante, cuando el glicerol es extraído dentro de la pajuela, la viabilidad embrionaria puede ser afectada si no se logra una mezcla homogénea de las distintas soluciones. El método se realiza de la siguiente manera:

1. Selección del embrión. Separar mórulas de blastocitos.

2. Lavar 10nveces con una solución de Dulbecco modificado, con $10 \%$ de suero fetal bovino.

3. Morulas: sumergir durante 10 minutos el embrión en una solución de Dulbecco, con 1,5 molar de glicerol, más 0,1 molar de sucrosa. Blastocistos: sumergir durante 10 minutos el 
embrión en una solución de Dulbecco, con 1,5 molar de glicerol, más 0,2 molar de sucrosa.

4. Tomar una pajuela de $0,25 \mathrm{cc}$ y cargar en ella el embrión en el siguiente orden:

a) 1ra. Columna de Dulbecco con suero fetal al 10\%.

b) 2da. Columna, pequeña burbuja de aire.

c) 3ra. Columna de Dulbecco con medio de congelación.

d) 4ta. Columna, pequeña burbuja de aire

e) 5 ta. Columna, cargar el embrión en su medio.

f) 6ta. Columna, pequeña burbuja de aire.

g) Completar el resto de la pajuela con medio de congelación.

5) Llevar los embriones a la congeladora con una temperatura de entre $-6^{\circ} \mathrm{C}$ a $-7^{\circ} \mathrm{C}$, donde permanecerán 10 minutos en stand by.

6) A su término tocar la pajuela a la altura de la 3ra. Columna con una pinza hemostática, previamente sumergida en nitrógeno líquido para que alcance su extremo los $-196^{\circ} \mathrm{C}$. este método (Seeding) evitara la muerte del embrión por superfusion.

7) La corrida de temperatura será de $0,5^{\circ} \mathrm{C}$ por minuto hasta alcanzar $\operatorname{los}-35^{\circ} \mathrm{C}$, momento en que se introducirá la pajuela en nitrógenos liquido (Pluging) (Ochoa, 2011).

\section{CRIOINJURIA}

El concepto de crioinjuria, se refiere al daño que sufren los embriones bovinos cuando son sometidos a bajas temperaturas durante el proceso de congelación-descongelación y está determinado por los siguientes puntos (Maurer, 1978; Niemann, 1985; Ávila-Portilla et al., 2006; Hincapie et al. 2005; Ochoa, 2011):

- Formación de hielo intra o extracelular.

- Aumento o disminución excesiva de volumen celular por efecto osmótico.

- Efecto tóxico del crioprotector.

- Alteraciones de las membranas celulares.

- Fractura embrionaria o de la zona pelúcida. 


\section{BIBLIOGRAFÍA}

Akyurt, M. Zaki, G. Habeebullah, B. 2002. Freezing phenomena in ice water systems. Energy Conversion and Management 43:1771-1789.

Ávila Portillo Luz Mabel, Madero José I., López Claudia, León María Fernanda, Acosta Lucía, Gómez Claudia, Delgado Lucy Gabriela, Gómez Claudio, Lozano José Manuel, Reguero María T. 2006. Fundamentos de criopreservación. Revista Colombiana de Obstetricia y Ginecología; 57 (4): 291-300.

Belascoain María Gabriela, Díaz Érica teresa y Hütr Soledad. 2010. Técnicas para la criopreservación de embriones bovinos. Trabajo final para optar al título de especialistas en reproducción bovina. Universidad Nacional de Córdoba. Facultad de ciencias agropecuarias escuela para graduados. Instituto de reproducción animal Córdoba.

Cabrera Pedro y Fernández Adriana. 2006. Criopreservación de embriones: una herramienta básica en la reproducción asistida. Rev Fac Cienc; 47 (2): 59-70.

Díez Monforte Carmen. 2003. Congelación de embriones bovinos producidos in vitro. Servicio Regional de Investigación y Desarrollo Agroalimentario. Sitio Argentino de Producción Animal: http://www.produccion-animal.com.ar/informacion_tecnica/transplante_embrionario/08congelacion_embriones.pdf

Joseph Saragusty and Amir Arav. 2011. Current progress in oocyte and embryo cryopreservation by slow freezing and vitrification. Reproduction Review: 141 1-19.

Maurer, R. 1978. Freezin of mammalian embryos: A review of the techniques. Theriogenology, 9: 45-68.

Mucci, N., Aller J. Cabodevila J., Kaiser G., Hozbor F. y Alberio R.H. 2005. Criopreservación de embriones bovinos. Taurus, Bs, As; 7 (26): 20-35.

Niemann, H. 1985. Freezing of bovine embryos: Effects of a one-step addition of 1,4M glycerol. Theriogenology; 23: 369-379. 
Noris Roa A., Tiburcio Linares y Rita Tamasaukas. 1998. Métodos y aplicación de la criopreservación de ovocitos y embriones en bovinos y otros mamíferos. Revista Científica, FCV-LUZ; 8 (1): 40-52.

Ochoa José Teodoro. 2011. Criopreservación de embriones bovinos. Curso de graduación "Buiatría" para obtención de título de Medicina veterinaria y zootecnia. Universidad de Cuenca. Facultad de ciencias agropecuarias. Escuela de medicina veterinaria y zootecnia.

Reggie G. Guerra M. 2011. Evaluación de tres protocolos de criopreservación de embriones bovinos obtenidos in vivo e in vitro. Trabajo de graduación sometido para optar por el título de master en Ciencias Pecuarias con énfasis en producción animal. Universidad de Panamá. Vicerrectoría de investigación y postgrado. Facultad de ciencias agropecuarias.

Rivas Caceres Raymundo y Barceló Fimbres Moises. 2011. Manuel de prácticas biología de la reproducción. Universidad Autonoma de ciudad Juárez. Instituto de Ciencias Biomédicas. Departamento de Ciencias Químico-Biológicas. Programa de biología.

Sánchez Muñoz José Bernardo, Mejía Villanueva Octavio y Manzur Cruz Alberto. 2014. Programa de transferencia de tecnología en aplicación de técnicas asistida para ovinos en el trópico. Manual de transferencia de embriones en ovinos. Tuxtla Gutiérrez Chiapas.

Stringfellow D.A. y Sidel Sarah M. 2000. Manual de la Sociedad Internacional de Transferencia de Embriones: Guía de procedimientos e información general para el us de la tecnología de la transferencia de embriones con especial énfasis. Editorial. International Embryo Transfer Society, 2000. $3^{\circ}$ Edición. 